Preprint of the paper

"Meshless methods for potential problems in electrical engineering applications" I. Colominas, G. Mosqueira, M. Chao, F. Navarrina, M. Casteleiro (1999)

En "Computational Methods in Engineering and Science", Volumen 1, Sección "FE and BE Methods", 381---392.

J. Bento, E. Arantes e Oliveira, E. Pereira (Editors); Elsevier Science Ltd., Oxford, UK. (ISBN: 08-043570-X-HC)

http://caminos.udc.es/gmni 


\title{
MESHLESS METHODS FOR POTENTIAL PROBLEMS IN ELECTRICAL ENGINEERING APPLICATIONS
}

\author{
I. Colominas, G. Mosqueira, M. Chao, F. Navarrina, \& M. Casteleiro \\ Dpto. de Métodos Matemáticos y Representación. Universidad de La Coruña \\ E.T.S. de Ingenieros de Caminos, Canales y Puertos \\ Campus de Elviña, 15071 La Coruña. SPAIN \\ Email: colominas@iccp.udc.es
}

\begin{abstract}
In some problems in engineering applications, the mesh generation process represents one of the big challenges when numerical methods such as finite elements, finite differences or boundary elements are applied. For this reason, several numerical techniques ("meshless methods") have been recently proposed to overcome the problems related with the discretization of the domain. These methods can represent an important improvement in Computational Mechanics, and among others in the electrical engineering field. In this paper, we present a meshless technique based on the Moving Least Square method with a point collocation approach for solving problems in Potential Theory in electrical engineering applications. Furthermore we propose the use of enrichment numerical approaches applied to these meshless procedures.
\end{abstract}

\section{KEYWORDS}

Meshless Methods, Enrichment functions, Moving Least Square Methods, Earthing

\section{INTRODUCTION}

Advances in numerical methods, together with the development of computer sciences, have represented a significant improvement in the treatment of some engineering problems. However, in some practical applications (e.g., problems with moving boundaries and discontinuities or in cases of domains with very complicated geometry) difficulties 
arise when standard numerical techniques which require the discretization of the whole domain are applied (Belytschko et al., 1998; Oñate et al., 1996-b). In fact, when an efficient grid is needed, the mesh generation process frequently becomes the bottle neck.

To overcome these problems, in recent years some different numerical techniques have been proposed, such as numerical methods where explicit element meshes are unnecessary ("meshless methods") (Belytschko et al., 1996) and numerical methods where approximations are enriched by using functions of the same type that the solution (Belytschko et al., 1998; Taylor et al., 1997).

In meshless methods, the solution domain is formed by a set of nodal points. Every point has an associated subdomain including its closest points and therefore, a local approximation can be achieved in each node. Thus, for every central node or "star node" this approximation is built with the information provided by its subdomain points. Within the limits of these techniques several methods have appeared in last years. One of them is based on least squares formulations combined with Point Collocation approaches to compute the integral terms, therefore no auxiliary grid is required. Furthermore, in this method, different interpolants can be derived depending on the weighting function: it can be fixed within each subdomain, as in the Diffuse Least Square Method (DLS), or it can depend on the point where the approximated value is to be computed, as in Moving Least Square Method (MLS) (Chao, 1997).

On the other hand, the essential idea of the use of "enrichment functions" is to improve the results obtained with other numerical methods by adding functions to base approximations which are a partition of unity. This last property corresponds to the ability of the shape functions to reproduce a constant, being crucial for convergence (Belytschko et al., 1998). Two kinds of enrichment have been proposed: the first one employs functions involved in the solution of the problem (Belytschko et al., 1998), and the other one proposes to enrich with polynomial functions (Taylor et al., 1995).

In this paper a moving least square approach using base interpolating functions normalized within domain, with a point collocation scheme it is proposed for solving potential problems in electrical engineering applications. Furthermore, the performance of this method combinated with enrichment functions is analyzed in some numerical tests.

\section{MOVING LEAST SQUARE APPROXIMATION}

Moving Least Square approximations lie in a local weighted least square fitting, valid on each collocation point subdomain formed by its $n$ closest nodal points (subdomain $\Omega_{k}$ ). The local character of the approximation comes from a moving weighting function which takes its maximum value at this collocation point and vanishes outside a surrounding region (Oñate et al., 1996-a).

The proper definition of the approximation at any point implies that all subdomains $\Omega_{k}$ cover all the interpolation domain. Hereby, these subdomains must overlap and the common areas have to include enough nodal points in order to ensure the convergence of the method. Thus, for a given collocation point the selection of the nodal points belonging to its subdomain has been performed according to an effective technique based on the "four quadrants" criterium (Oñate et al., 1996-a).

Let $\Omega_{k}$ be the interpolation domain of a function $u(\boldsymbol{x})$. A local approximation to $u(\boldsymbol{x})$ 
can be derived in the form,

$$
u(\boldsymbol{x}) \cong \hat{u}(\boldsymbol{x})=\sum_{i=1}^{m} p_{i}(\boldsymbol{x}) \alpha_{i}=\boldsymbol{p}^{t}(\boldsymbol{x}) \boldsymbol{\alpha}
$$

where $\boldsymbol{\alpha}=\left[\alpha_{1}, \alpha_{2}, \ldots, \alpha_{m}\right]^{t}$ is a set of unknown coefficients and $\boldsymbol{p}(\boldsymbol{x})$ contains a base of interpolating functions (monomial terms, generally) which order is $m$ (Oñate et al., 1996-b). These base interpolating functions can be normalized within each subdomain $\Omega_{k}$ dividing by the maximum distance $d$ between the collocation point $i$ and the most distant point of its subdomain. The normalized coordinates are:

$$
\boldsymbol{\xi}(\boldsymbol{x})=\left[\frac{x-x_{i}}{d}, \frac{y-y_{i}}{d}, \frac{z-z_{i}}{d}\right]
$$

On the other hand, function $u(\boldsymbol{x})$ can be sampled in the $n$ nodal points belonging to $\Omega_{k}$ in the form,

$$
\boldsymbol{u}^{h}=\left(\begin{array}{c}
u_{1}^{h} \\
\vdots \\
u_{n}^{h}
\end{array}\right) \cong\left(\begin{array}{c}
\hat{u}_{1} \\
\vdots \\
\hat{u}_{n}
\end{array}\right)=\left(\begin{array}{c}
\boldsymbol{p}_{1}^{t} \\
\vdots \\
\boldsymbol{p}_{n}^{t}
\end{array}\right) \boldsymbol{\alpha}=\boldsymbol{S} \boldsymbol{\alpha}
$$

being $u_{j}^{h}$ the values of unknown function evaluated in nodal points of subdomain $\Omega_{k}$ $\left(u_{j}^{h}=u\left(\boldsymbol{x}_{j}\right), j=1, \ldots, n\right), \hat{u}_{j}=\hat{u}\left(\boldsymbol{x}_{j}\right)$ their approximated values, and $\boldsymbol{p}_{j}$ contains the normalized base interpolating functions evaluated in $\boldsymbol{\xi}_{j}$ (where $\boldsymbol{\xi}_{j}=\boldsymbol{\xi}\left(\boldsymbol{x}_{j}\right)$ ).

In general, the number of nodal points $n$ is greater than the order $m$ of the polinomials base, so $\boldsymbol{S}$ is a rectangular matrix and the approximation cannot fit all the $u_{j}^{h}$ values. However, approximated values $\hat{u}(\boldsymbol{x})$ can be determined by minimizing the weighted sum of the square differences between the exact value $u_{j}^{h}$ and the approximation $\hat{u}\left(\boldsymbol{x}_{j}\right)$ at each nodal point $\boldsymbol{x}_{j}$ belonging to the domain of the arbitrary collocation point $\boldsymbol{x}_{k}$ (Oñate et $a l ., 1996-\mathrm{b})$. The weighting function in moving least square approximation computed in $\boldsymbol{x}_{j}, \omega_{k}\left(\boldsymbol{x}_{j}, \boldsymbol{x}_{k}\right)$, is usually built in such a way that it equals unity in collocation point $\boldsymbol{x}_{k}$ and vanishes outside domain $\Omega_{k}$. In the examples presented in this paper, the truncated gaussian distribution has been used.

In general, the definition of a different weighting function for every interpolating point $\boldsymbol{x}_{k}$ is very difficult, presenting an infinite number of posibilities. To overcome this problem, we can define the weighting functions at nodal points $\boldsymbol{x}_{j}$, and use them evaluated in the arbitrary point $\boldsymbol{x}_{k}$. Therefore, $\omega_{k}\left(\boldsymbol{x}_{j}, \boldsymbol{x}_{k}\right)$ may be substituted by $\omega_{j}\left(\boldsymbol{x}_{j}, \boldsymbol{x}_{k}\right)$. To simplify the notation $\boldsymbol{x}_{k}$ is replaced by the global coordinate $\boldsymbol{x}$.

Thus, the functional to be minimized with respect to $\boldsymbol{\alpha}$ results in

$$
J(\boldsymbol{x})=\sum_{j=1}^{n} \omega_{j}\left(\boldsymbol{x}_{j}, \boldsymbol{x}\right)\left(u_{j}^{h}-\hat{u}\left(\boldsymbol{x}_{j}\right)\right)^{2}
$$

The performance of this process yields to (Chao, 1997),

$$
\boldsymbol{\alpha}=\boldsymbol{A}^{-1}(\boldsymbol{x}) \boldsymbol{B}(\boldsymbol{x}) \boldsymbol{u}^{h} \quad \boldsymbol{A}(\boldsymbol{x})=\boldsymbol{P} \boldsymbol{W}(\boldsymbol{x}) \boldsymbol{P}^{t} \quad \boldsymbol{B}(\boldsymbol{x})=\boldsymbol{P} \boldsymbol{W}(\boldsymbol{x})
$$


being auxiliary matrices $\boldsymbol{P}$ and $\boldsymbol{W}(\boldsymbol{x})$ :

$$
\boldsymbol{P}=\left[\begin{array}{llll}
\boldsymbol{p}\left(\boldsymbol{\xi}_{1}\right) & \ldots & \left.\boldsymbol{p}\left(\boldsymbol{\xi}_{n}\right)\right]
\end{array} \quad \boldsymbol{W}(\boldsymbol{x})=\operatorname{diag}\left[\omega_{k}\left(\boldsymbol{x}_{j}, \boldsymbol{x}\right)\right], \quad j=1, \ldots, n\right.
$$

Substitution of (5) in (1) allows to obtain an approximation to function $u(\boldsymbol{x})$ in $\Omega_{k}$ :

$$
u(\boldsymbol{x}) \cong \hat{u}(\boldsymbol{x})=\boldsymbol{p}^{t}(\boldsymbol{\xi}) \boldsymbol{A}^{-1}(\boldsymbol{x}) \boldsymbol{B}(\boldsymbol{x}) \boldsymbol{u}^{h} .
$$

Therefore, one can define the shape functions in $\boldsymbol{x}$ as [4],

$$
\boldsymbol{N}^{t}(\boldsymbol{x})=\boldsymbol{p}^{t}(\boldsymbol{\xi}) \boldsymbol{A}^{-1}(\boldsymbol{x}) \boldsymbol{B}(\boldsymbol{x})
$$

and consequently,

$$
\hat{u}(\boldsymbol{x})=\sum_{j=1}^{n} N_{j}(\boldsymbol{x}) u_{j}^{h}
$$

It must be taken into account that the local values of the approximating function do not fit the nodal unknown values, $\hat{u}\left(\boldsymbol{x}_{j}\right) \neq u_{j}^{h}$, due to the least square character of the approximation. It must also be pointed out that if $n=m$, the FEM type approximation is recovered and no effect of weighting is presented (Oñate et al., 1996-a). Besides, if the weighting function is constant and equals the unity, the standard least square method is reproduced.

\section{MLS APPROXIMATIONS WITH ENRICHMENT FUNCTIONS}

The introduction of enrichment functions to moving least square approachs consists of adding to the approximation function (9) new terms which contain information about the solution, in order to decrease the global computacional cost. Thus, when the kind of functions in the solution is known, the application of this idea can be very useful. In this case, the approximation takes the form of an extrinsic enrichment (Belystchko et al., 1998) and can be written as:

$$
u(\boldsymbol{x}) \cong \hat{u}(\boldsymbol{x})=\sum_{j=1}^{n} N_{j}(\boldsymbol{x})\left(u_{j}^{h}+\sum_{k=1}^{n_{f}} a_{k j} F_{k}(\boldsymbol{x})\right)
$$

where $\hat{u}$ is the approximation to the exact solution $u$ in the domain $\Omega_{k}, n_{f}$ is the total number of enrichment functions $F_{k}$, and $n$ is the total number of nodal points. The approximation to the solution requires to know in every one of the $n$ nodal points the value of $n_{f}+1$ coefficients: $\boldsymbol{u}_{j}$ and $\boldsymbol{a}_{k, j}$. For this reason when the MLS approximation is combined with a point collocation approach, it requires $\left(n_{f}+1\right) n$ collocation points.

One important property, in order to study the convergence of this method, is that as $N_{j}(\boldsymbol{x})$ is obtained in the same way as in MLS formulation, the partition of unity is satisfied. Therefore, a hierarchical enrichment may be added using any type of functions (Taylor et al., 1997).

This idea of the enrichment has been successfully applied in Finite Elements and Element Free Galerkin Methods in elasticity problems and fracture mechanics problems (Belystchko et al., 1998; Taylor et al., 1997). In the same way, in this paper we propose the use of enriched meshless formulations (EMF) in some applications in electrical engineering. 


\section{A BOUNDARY PROBLEM DISCRETIZED EQUATIONS}

In order to study applications of preceding methods, in this section are stated the discretized equations for a boundary problem.

Let $A$ and $B$ be two differential operators, $\Omega$ the domain of our problem and $\Gamma$ its boundary $\left(\Gamma=\Gamma_{t} \cup \Gamma_{u}\right)$. In these terms, a scalar boundary value problem can be written as,

$$
A(u)=b \quad \text { in } \quad \Omega
$$

with boundary conditions,

$$
\begin{array}{ccc}
B(u)=t & \text { in } \quad \Gamma_{\mathrm{t}} \\
u-u_{p}=0 & \text { in } & \Gamma_{\mathrm{u}}
\end{array}
$$

where $u$ is the solution, $b$ and $t$ represent the actions over $\Omega$ and along the boundary $\Gamma_{t}$, and $u_{p}$ is the prescribed value of $u$ along $\Gamma_{u}$.

Application of weighted residual method allows to obtain a variational form of the above problem, in terms of the trial approximation function $\hat{u}$ of the unknown $u$, as

$$
\int_{\Omega} \Psi_{i}[A(\hat{u})-b] d \Omega+\int_{\Gamma_{t}} \widehat{\Psi}_{i}[B(\hat{u})-t] d \Gamma+\int_{\Gamma_{u}} \widehat{\widehat{\Psi}}_{i}\left[\hat{u}-u_{p}\right] d \Gamma=0
$$

which must hold for all members $\Psi_{i}, \widehat{\Psi}_{i}$ and $\widehat{\hat{\Psi}}_{i}$ of suitable classes of test functions defined on $\Omega, \Gamma_{t}$ and $\Gamma_{u}$. The selection of test functions in the general varational form (13) allows to derive different formulations. In the examples presented in this paper a point collocation method has been implemented, in order to take advantage of the meshless character of the approximation (Chao, 1997). Other approaches based on integral methods have been proposed (Oñate et al., 1996-a; Taylor et al., 1997), but require some kind of auxiliar grid to evaluate the resulting integrals.

The point collocation scheme $\left(\Psi_{i}=\widehat{\Psi}_{i}=\widehat{\widehat{\Psi}}_{i}=\delta_{i}\right.$ in expression (13), where $\delta_{i}$ is the Dirac delta) leads to the set of equations,

$$
\begin{array}{rll}
{[A(\hat{u})]_{i}-b_{i}=0} & \text { in } & \Omega \\
{[B(\hat{u})]_{i}-t_{i}=0} & \text { in } & \Gamma_{t} \\
\hat{u}_{i}-u_{p}=0 & \text { in } & \Gamma_{u}
\end{array}
$$

If function $\hat{u}$ is approximated by linear combination of the shape functions $(9,10)$ the previous system of equations may be written in the standard form as,

$$
\boldsymbol{K} \boldsymbol{u}^{h}=\boldsymbol{f}
$$

where coefficient matrix $\boldsymbol{K}$ is banded (but not necessary symmetric), $\boldsymbol{f}$ is also known (contains the contributions from terms $b$ and $t$ and prescribed values $u_{p}$ ), and $\boldsymbol{u}^{h}$ contains the unknown values of the function evaluated in nodal points (Chao, 1997). 


\section{NUMERICAL TESTS}

In this section we present two numerical tests of the MLS approach applied to the solution of a 1D boundary value problem. Thus, it will be compared results obtained by using a standard MLS and an enrichment MLS approach.

\section{Example 1}

As a first example we consider the following BVP (Carey et al., 1997):

$$
-\frac{d^{2} u}{d x^{2}}+u=f(x), \quad 0 \leq x \leq 1, \quad u(0)=0, \quad u(1)=1
$$

being

$$
f(x)=\frac{2 \rho\left[1+\rho^{2}(1-\beta)(x-\beta)\right]}{\left[1+\rho^{2}(x-\beta)^{2}\right]^{2}}+(1-x)[\operatorname{atan}(\rho(x-\beta))+\operatorname{atan}(\rho \beta)]
$$

The analytical solution is given by

$$
u(x)=(1-x)[\operatorname{atan}(\rho(x-\beta))+\operatorname{atan}(\rho \beta)]
$$

Depending on the election of parameters $\rho$ and $\beta$ in (17), we can control the shape of the solution. In this case $\rho=50.0, \beta=0.4$ produce a sharp function, that it is very difficult to approximate, being a good test for the performance of the method. Thus, we can approximate the solution to (16) and (17) with a meshless method for a given set of $n_{p}$ trial functions $N_{i}$ defined on the domain, by using one enrichment function $\left(F_{1}(x)=\operatorname{atan}(x)\right)$ in $(10)$. In Figure 1 it is shown a comparison between the analytical solution and the MLS approximations with and without enrichment.

\section{Example 2}

As a second example, we consider the BVP that represents a string on an elastic foundation (Taylor et al., 1995):

$$
-a \frac{d^{2} u}{d x^{2}}+c u=f, \quad 0 \leq x \leq 1, \quad u(0)=0, \quad u(1)=1
$$

The analytical solution is given by:

$$
u(x)=\frac{f}{c}\left[1-\cosh (x / \sqrt{a})-(1-\cosh (1 / \sqrt{a})) \frac{\sinh (x / \sqrt{a})}{\sinh (1 / \sqrt{a})}\right]
$$

Parameters in (19) are given by $a=0.01$ and $c=f=1$. In this case, the enrichment meshless approximation to this problem has been obtained by using two enrichment functions $F_{1}(x)=\cosh (x)$ and $F_{2}(x)=\sinh (x)$ in (10). In Figure 2 it is shown a comparison between the analytical solution and the approximations obtained.

In the light of these $1 \mathrm{D}$ test examples it can be noticed that results obtained by using meshless methods can improve if enrichment functions are used. At present, we are working in this way in order to introduce enrichment functions in meshless approximations in $2 \mathrm{D}$ and $3 \mathrm{D}$ problems. In the following section we present an application to grounding analysis of the MLS method. 


\section{APPLICATION TO GROUNDING ANALYSIS}

Grounding systems in substations has to guarantee the integrity of equipments and the continuity of the service under fault conditions - providing means to carry and dissipate electrical currents into the ground-, and to safeguard that persons in the surroundings of the grounded installation are not exposed to dangerous electrical shocks. To achieve these goals, the equivalent electrical resistance of the system must be low enough to assure that fault currents dissipate mainly through the grounding grid into the earth, while maximum potencial differences between close points on the earth surface must be kept under certain tolerances (step, touch and mesh voltages).

Physical phenomena underlying fault currents dissipation into the earth can be modelled by means of Maxwell's Electromagnetic Theory (Colominas et al., 1997). Constraining analysis to the obtention of the electrokinetic steady-state response, and neglecting the inner resistivity of the earthing electrode, the $3 \mathrm{D}$ problem associated to an electrical current derivation to earth can be written as

$$
\begin{gathered}
\operatorname{div} \boldsymbol{\sigma}=0, \quad \boldsymbol{\sigma}=-\underline{\underline{\boldsymbol{\gamma}}} \operatorname{grad} V \text { in } E \\
\boldsymbol{\sigma}^{t} \boldsymbol{n}_{E}=0 \text { in } \Gamma_{E} ; \quad V=V_{\Gamma} \text { in } \bar{\Gamma} ; \quad V \longrightarrow 0, \text { if }|\boldsymbol{x}| \rightarrow \infty ;
\end{gathered}
$$

where $E$ is the earth, $\boldsymbol{\gamma}$ its conductivity tensor, $\Gamma_{E}$ the earth surface, $\boldsymbol{n}_{E}$ its normal exterior unit field and $\overline{\bar{\Gamma}}$ the electrode surface (Belystchko et al., 1996). Thus, when the electrode attains a voltage $V_{\Gamma}$ (Ground Potential Rise or GPR) relative to a distant grounding point, the solution to this problem gives the potential $V$ and the current density $\boldsymbol{\sigma}$ at an arbitrary point $\boldsymbol{x}$. Further assumption $V_{\Gamma}=1$ is not restrictive at all, since $V$ and $\boldsymbol{\sigma}$ are proportional to $V_{\Gamma}$.

The example presented in this paper consists of a toroidal electrode horizontally buried to a depth of $7 \mathrm{~m}$. The interior diameter of the ring is $20 \mathrm{~m}$ and the electrode diameter is $3 \mathrm{~m}$. The upper layer depth is $14 \mathrm{~m}$. The scalar conductivity associated with the lower layer $\gamma_{2}$, is four times the one corresponding to the upper layer $\gamma_{1}\left(\gamma_{2}=4 \gamma_{1}\right)$. Due to the axial symmetry of the problem, solution can be obtained by using a 2D model. This case has been solved with 3019 points obtained by means of the program GEN4U (Sarrate, 1996). The base interpolating functions used are linear and all subdomains contain at least five points. Figure 3 shows the nodal point distribution, the contour lines and the potential distribution around the electrode in two cases: assuming the hypothesis of homegeneous and isotropic soil or considering a two-layer model. These numerical results agree significantly with those obtained by using a very dense point distribution and with results of a boundary element program.

\section{CONCLUSIONS}

In this paper, a moving least square interpolation method with a point collocation approach using enrichment functions has been presented. This technique has been compared for $1 \mathrm{D}$ numerical tests with the analytical solution and the MLS method without enrichment. Results significantly agree and very good results are obtained when enrichment functions are used. 
Furthermore the MLS approach has been applied to the solution of a problem in grounding analysis. First results obtained for different point distributions, even with a two-layer soil model, are very promising and require a reasonable computational cost. Obviously, further analysis must be done in both mathematical and numerical aspects in order to introduce the enrichment procedure to $2 \mathrm{D}$ and $3 \mathrm{D}$ problems.

\section{ACKNOWLEDGEMENTS}

This work has been partially supported by the "Subdirección General de Proyectos de Investigación Científica y Técnica (SGPICYT) del Ministerio de Educación y Cultura (1FD97-0108)", cofinanced with FEDER funds and the power company "Unión Fenosa Ingeniería S.A. (UFISA)", and by research fellowships of the "Secretaría General de I + D de la Xunta de Galicia" and the "Universidad de La Coruña".

\section{REFERENCES}

Belystchko T., Krongauz Y., Organ D., Krysl P. (1996): "Meshless methods: An overview and recent developments", CMAME, 139, 3-48.

Belystchko T., Black T. (1998): "Elastic Crack Growth in Finite Elements with Minimal Remeshing", Research Report of the TAM Group, Northwestern University.

Carey G.F., Oden J.T. (1983): "Finite elements", Prentice-Hall Inc., vol II, New Jersey.

Chao M. (1997): "Introducción a los Métodos Numéricos sin Malla: fundamentación y desarrollo de formulaciones de minimos cuadrados ponderados", Research Report. Universidad de La Coruña.

Colominas I., Chao M., Navarrina F., Casteleiro M. (1998): "Application of Meshless Methods to the analysis and design of grounding systems", In "Computational Mechanics: New Trends and Appl.", S. Idelsohn, E.Oñate, Dvorkin Ed.; CIMNE, Barcelona.

Colominas I., Aneiros J., Navarrina F., Casteleiro M. (1997): "A Boundary Element Numerical Approach for Substation Grounding in a Two Layer Earth Structure", Advances in Computational Engineering Science, Section "Recent Developments in Boundary Element Method", 756-761, S.N. Atluri, G. Yagawa Ed.; Tech Science Press, Atlanta.

Oñate E., Idelsohn S., Zienkiewicz O.C., Taylor R.L. (1996-a): “A finite point method in computational mechanics. Applications to convective transport and fluid flow", IJNME, 39, 3839-3867.

Oñate E., Idelsohn S., Zienkiewicz O.C., Taylor R.L., Sacco C. (1996-b): “A stabilized finite point method for analysis of fluid mechanics problems", CMAME,139,315-346.

Sarrate J. (1996): "Modelización numérica de la interacción fluido-sólido rígido: Desarrollo de algoritmos, generación de mallas y adptabilidad", $\mathrm{PhD}$ Thesis, UPC, Barcelona.

Taylor R.L., Idelsohn S., Zienkiewicz O.C., Oñate E. (1995): "Moving Least Square Approximation for solution of differential equations", No. 74, CIMNE, Barcelona.

Taylor R.L., Zienkiewicz O.C., Oñate E. (1997): "A hierarchical finite element method based on the partition of unity", CMAME, 152, 73-84. 


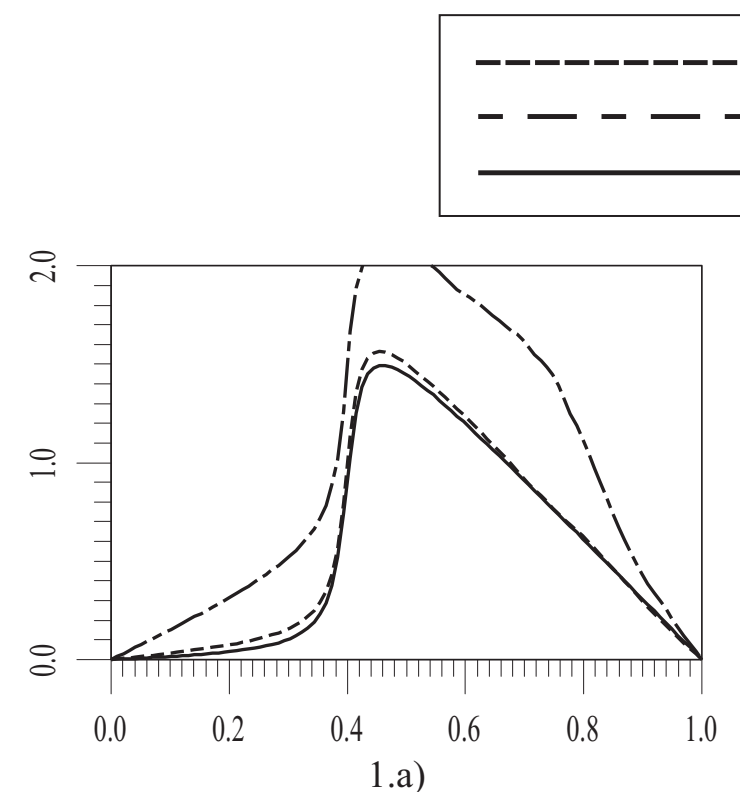

MLS with enrichment

MLS

Analytical Solution
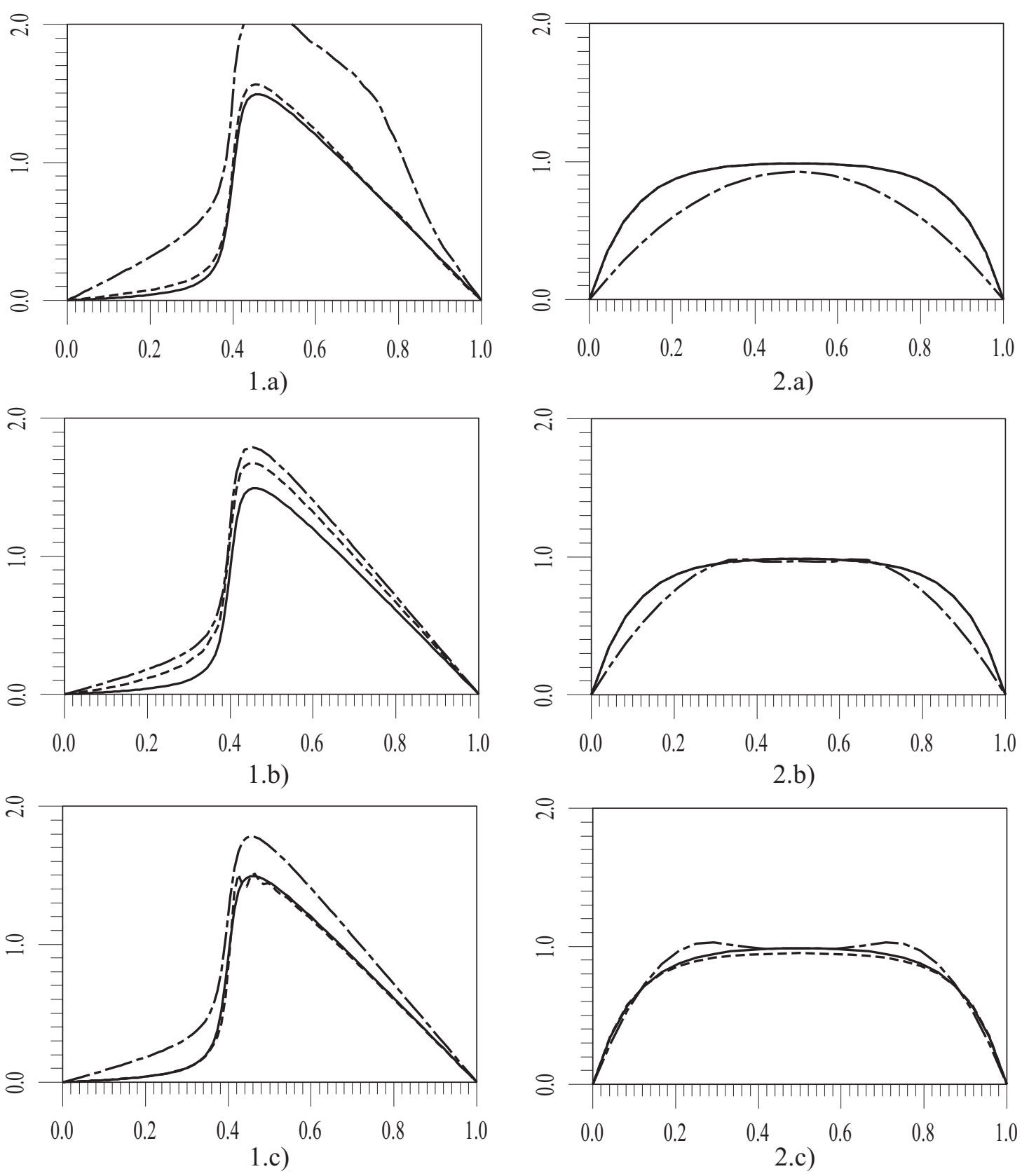

Fig. 1.- Example 1: Comparison of results ob- Fig. 2.- Example 2: Comparison of results obtained by using an enriched MLS approach, a standard MLS approach (both with quadratic interpolation), and the analytical solution: a) 50 nodal points, subdomains of 3 points; b) 50 nodal points, subdomains of 5 points; c) 50 nodal points, subdomains of 7 points

tained by using an enriched MLS approach, a standard MLS approach (both with quadratic interpolation), and the analytical solution: a) 3 nodal points, subdomains of 3 points; b) 5 nodal points, subdomains of 5 points; c) 7 nodal points, subdomains of 7 points 

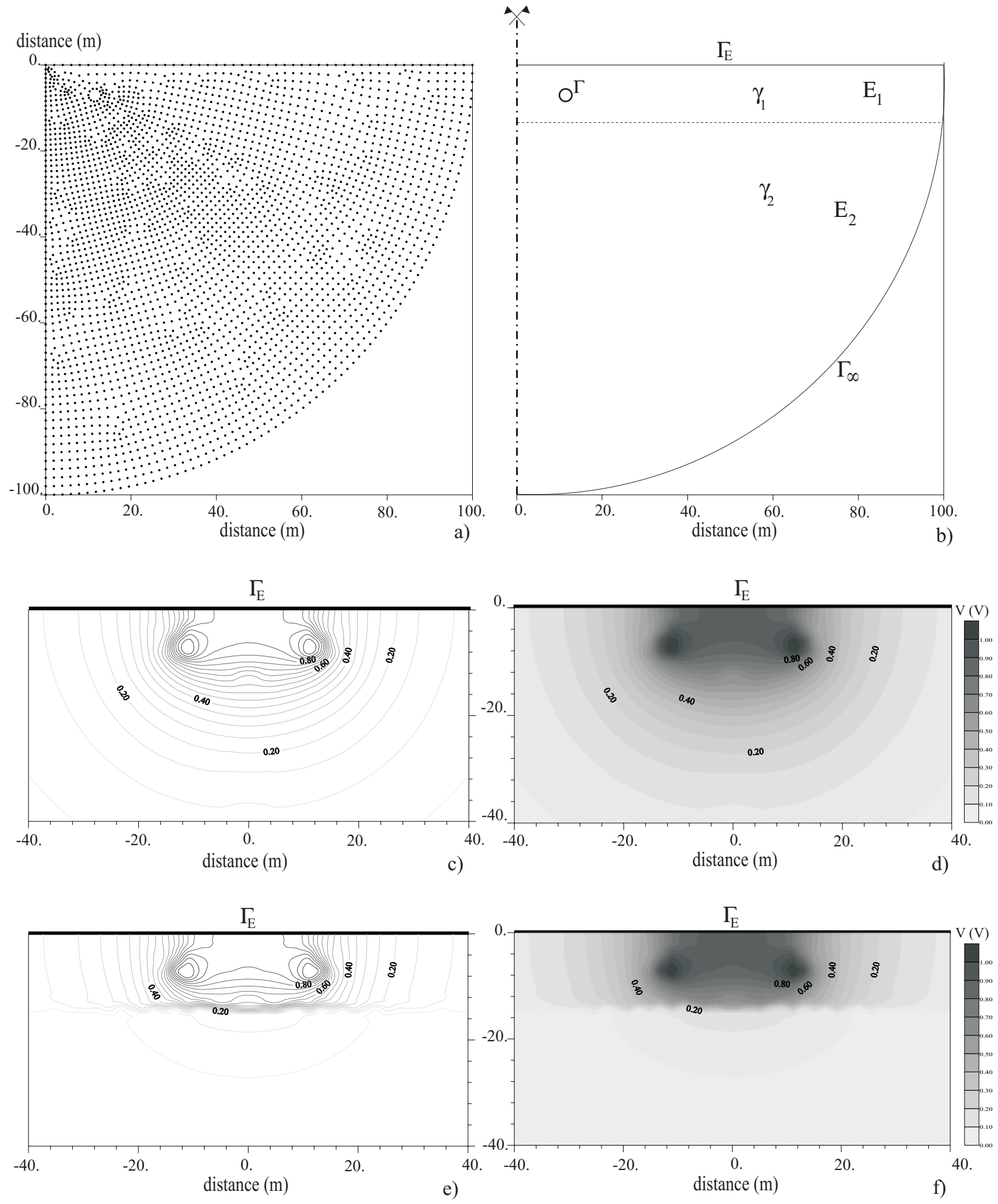

Figure 3.- Toroidal electrode buried to a depth of $7 \mathrm{~m}$ : a) Distribution of nodal points (npoin=3019), b) Domain solution scheme, c) Contour lines around the electrode, considering the soil homogeneous and isotropic, d) Potential distribution around the electrode, considering the soil as homogeneous and isotropic, e) Contour lines around the electrode assuming a two- layer model $\left(\gamma_{2} / \gamma_{1}=4\right)$, f) Potential distribution around the electrode assuming a two-layer model $\left(\gamma_{2} / \gamma_{1}=4\right)$. 